\title{
Charge control in laterally coupled double quantum dots
}

\author{
G. Muñoz-Matutano, ${ }^{1}$ M. Royo, ${ }^{2}$ J. I. Climente, ${ }^{2}$ J. Canet-Ferrer, ${ }^{1}$ D. Fuster, ${ }^{1}$ P. Alonso-González, ${ }^{3}$ I. Fernández-Martínez, ${ }^{3}$ \\ J. Martínez-Pastor, ${ }^{1}$ Y. González, ${ }^{3}$ L. González, ${ }^{3}$ F. Briones, ${ }^{3}$ and B. Alén ${ }^{3, *}$ \\ ${ }^{1}$ ICMUV, Instituto de Ciencia de Materiales, Universidad de Valencia, P.O. Box 22085, E-46071 Valencia, Spain \\ ${ }^{2}$ Departament de Química Física i Analítica, Universitat Jaume I, Castellón, Spain \\ ${ }^{3}$ IMM, Instituto de Microelectrónica de Madrid (CNM, CSIC), Isaac Newton 8, 28760 Tres Cantos, Madrid, Spain
}

(Received 27 April 2011; published 27 July 2011)

\begin{abstract}
We investigate the electronic and optical properties of InAs double quantum dots grown on GaAs (001) and laterally aligned along the [110] crystal direction. The emission spectrum has been investigated as a function of a lateral electric field applied along the quantum dot pair mutual axis. The number of confined electrons can be controlled with the external bias, leading to sharp energy shifts which we use to identify the emission from neutral and charged exciton complexes. Quantum tunneling of these electrons is proposed to explain the reversed ordering of the trion emission lines as compared to that of excitons in our system.
\end{abstract}

DOI: 10.1103/PhysRevB.84.041308

PACS number(s): 78.67.Hc, 73.21.La, 78.55.Cr

Optical spin initialization of individual electrons is a fundamental resource for quantum information science which relies on our ability to control the charge in a quantum dot (QD) molecule. ${ }^{1-3}$ In the past, vertically aligned QDs have been fabricated with great success. In these systems, exciton coupling signatures including energy anticrossings of neutral and charged exciton complexes, have been demonstrated by applying an electric field in the growth direction. ${ }^{4-6}$ Lateral QD molecules would be a better candidate for scaling up the electronic coupling from two to several QDs by applying individual lateral gates. Previous demonstrations of electronic coupling in a lateral QD pair have been based on an analysis of the anomalous Stark shifts and photon correlation statistics of the neutral exciton under a lateral electric field. ${ }^{7}$ Yet, the observation of electrically tunable energy anticrossings in lateral QD molecules remains a difficult task due to the exponential decrease of the tunnel coupling energy with the center-to-center QD distance $d .^{7-9}$ In the following, we investigate the emission spectrum of electrically tunable lateral QD pairs with a varying number of electrons. For typical interdot distances $d \sim 30-40 \mathrm{~nm}$, we find that electron tunneling phenomena affect the negative trion emission energy before clear exciton anticrossings may take place.

For the present study, QD pairs aligned in the [110] crystal direction were fabricated on GaAs nanoholes using a modified droplet epitaxy growth procedure as described in detail elsewhere. ${ }^{10}$ The nanostructures were grown on a $0.5-\mu \mathrm{m}$-thick undoped GaAs buffer layer and capped by $100 \mathrm{~nm}$ of undoped GaAs. Atomic force micrographs (AFMs) performed on a similar uncapped sample revealed that each QD in the pair has a slightly different height, with average values of $5.3 \pm 0.9$ and $6.6 \pm 1.5 \mathrm{~nm}$, respectively, and centerto-center separation equal to their average diameter $37 \pm 4 \mathrm{~nm}$ [Fig. 1(a)]. The morphological analysis also revealed that QD pair formation occurs in $95 \%$ of the cases with a small probability for single QDs or empty nanoholes. The low areal density of $2 \times 10^{8} \mathrm{~cm}^{-2}$ is adequate to study individual quantum nanostructures.

To apply an electric field along the QD pair mutual axis, we defined metal-semiconductor-metal (MSM) diodes by evaporation of two metal contacts $(15 \mathrm{~nm} \mathrm{Mo}+30 \mathrm{~nm} \mathrm{Au})$ on top of $100-\mu \mathrm{m}$-square mesas. The contacts are separated by a $80 \mu \mathrm{m}$ long $\times 1.5 \mu \mathrm{m}$ wide undoped GaAs channel embedding the nanostructures as shown in Figs. 1(a)-1(c). The microphotoluminescence $(\mu$-PL) of individual QD pairs was collected at $5 \mathrm{~K}$ using a fiber-based confocal microscope, excited with 785-nm continuous-wave laser light, dispersed by a $2 \times 0.3 \mathrm{~m}$ focal length double spectrograph and detected with a peltier cooled silicon charge coupled device (CCD) camera. The spectral resolution of our setup is $\sim 90 \mu \mathrm{eV}$ full width at half maximum (FWHM).

In the past few years, several groups have investigated the emission of single semiconductor nanostructures in the presence of a lateral electric field. For moderate electric fields, or when the separation between the contacts is large, the changes observed in the QD spectrum have been related to the modulation of the carrier capture probability induced by the external field. ${ }^{11,12}$ The capture mechanisms also play the most important role in single QDs dynamically driven by surface acoustic waves. ${ }^{13,14}$ The laterally applied bias can also modulate the electronic confinement levels. This requires larger electric fields or smaller contact separation for a given bias range. In this regime, the exciton wave function can be directly modified, leading to energy shifts, carrier tunneling, and fine-structure splitting reduction, among other effects. ${ }^{15-19}$ With a channel width of only $1.5 \mu \mathrm{m}$, our MSM diodes have been designed to apply large electric fields in the [110] crystal direction $(0-60 \mathrm{kV} / \mathrm{cm})$. This is required to tune independently the exciton energy of the two QDs in a lateral molecule and, if their separation were small enough, to observe resonant quantum tunneling phenomena.

The contour maps in Figs. 1(d) to 1(f) show the evolution of the $\mu$-PL spectrum as a function of the lateral bias $\Delta V$ for four different nanostructures (QN1-QN4). In each case, the evolution of the spectrally integrated intensity is also drawn [orange (spotted) lines]. The integrated intensity remains constant within $10 \%$ for a broad voltage range centred at $\Delta V_{c} \neq 0 \mathrm{~V}$ and diminishes down to zero for larger positive or negative bias. While the intensity decreases, the emission spectrum also changes, giving rise to blueshifted spectral features which are different in each nanostructure, as shown in the $\mu$-PL contour maps. These emission patterns can 


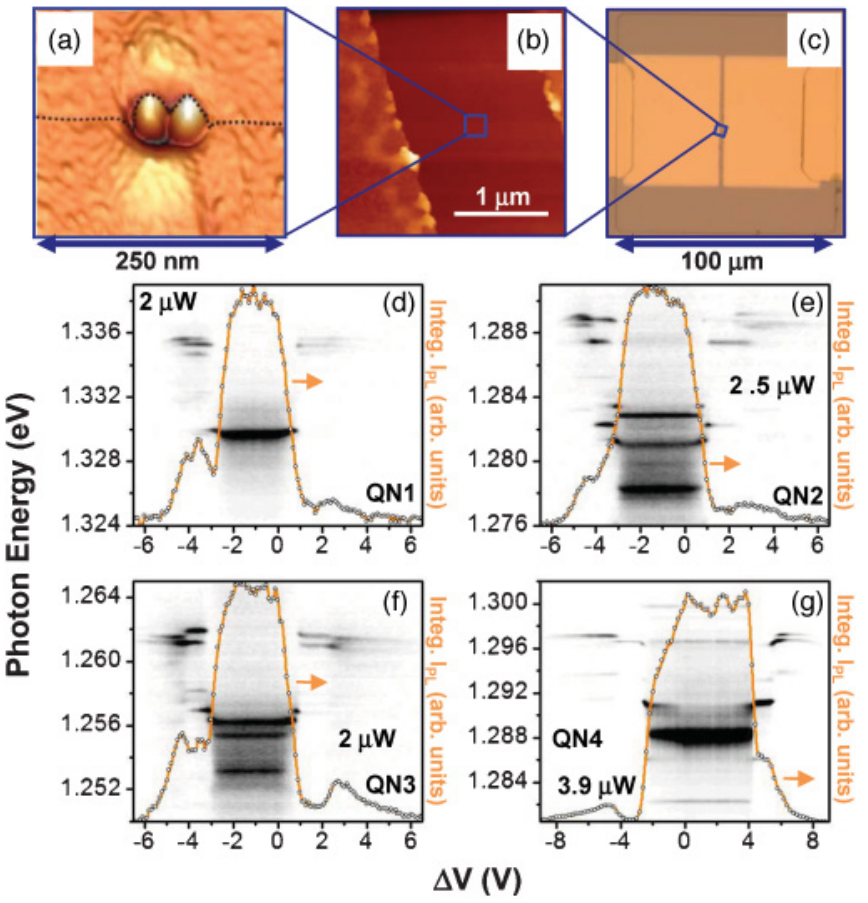

FIG. 1. (Color online) (a), (b), and (c) show two AFM images and an optical image of a single QD pair, a 1.5- $\mu$ m-wide GaAs channel, and a full MSM device, respectively. (d)-(f) show contour maps of the bias-dependent $\mu$-PL measured in four different nanostructures. The orange (spotted) lines stand for the $\mu$-PL intensity integrated in the corresponding emission ranges.

be examined to distinguish between single quantum dots (QN1) and double quantum dots (QN2-QN4) as exemplarily explained below for QN1 and QN2.

To do so, we have analyzed the device operation using semianalytical transport equations valid for one-dimensional (1D) MSM structures. ${ }^{20}$ The simulations are performed in dark conditions and explain why the spectrum is not fully symmetric with respect to $\Delta V=0 \mathrm{~V}$. Attending to the particular position of the nanostructure within the GaAs channel, the electric field is calculated, neglecting possible screening effects induced by the photogenerated carriers. Figure 2(a) shows the $E(\Delta V)$ dependence (black solid line) at the position of QN1, which we estimate according to the model at $\sim 530 \mathrm{~nm}$ from the left-hand contact. The curve shows a plateau which extends over the bias region where the field is still zero at this position. In this region, the integrated intensity [orange (spotted) line] and the $\mu$-PL spectrum [contour plot map in Fig. 2(b)] is independent of the voltage and, in the case of $\mathrm{QN} 1$, is characterized by a single broad resonance at $1.330 \mathrm{eV}$ (labeled as P1). As we approach the edges of the plateau raising $|\Delta V|$, the electric field in the vicinity of the nanostructure also increases. In such a situation, the possibility of an enhanced capture of carriers driven by the external lateral field was discussed by Moskalenko et al. ${ }^{12}$ They found that the overall QD integrated intensity increased rather than decreased for both positive and negative bias, and they also reported switching between spectral lines. The latter was explained by the uneven capture of electron and holes, and was found to be strongly dependent on the excitation energy and power and also on the temperature. In our case, we observe

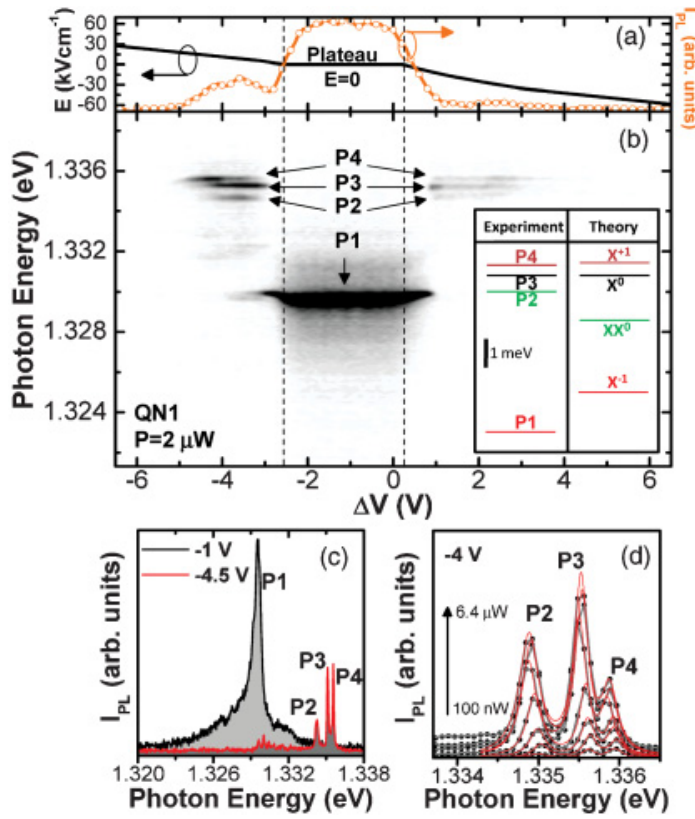

FIG. 2. (Color online) (a) Electric field vs bias dependence calculated using a semianalytical transport model (black solid line). Spectrally integrated $\mu$-PL intensity obtained in the same bias range [orange (spotted) line]. (b) $\mu$-PL contour plot map vs lateral bias measured for QN1. The inset compares the experimental (left-hand side) and theoretical (right-hand side) emission energies for lines P1-P4. (c) Two spectra extracted from the contour plot at the indicated voltages. (d) Excitation power evolution of P1-P4 and corresponding Lorentzian fits (solid lines).

an overall reduction of the integrated intensity and switching between spectral lines which are similar in a wide range of excitation powers and temperatures..$^{20}$ Our observations should be thus related to the large electric fields present in our devices and to intrinsic properties of InAs QDs and QD pairs grown by modified droplet epitaxy.

The decrease of the integrated intensity at both sides of the central plateau might be explained through a combination of two effects. First, electron and holes photogenerated above the barrier can drift away from the illuminated area, before being captured in the QDs, contributing to the subnanosecond photocurrent response of MSM photodetectors of this size. ${ }^{21}$ Second, carriers already confined in the nanostructure can tunnel out due to the large applied field. The latter causes the switching of spectral lines which we associate with the recombination of exciton complexes with a varying number of electrons. ${ }^{22}$ If the crystallization of the Ga droplet is not complete, arsenic vacancies arise during growth, creating localized states in the gap. These localized states are close in energy to the electron confined levels and are occupied by one or more electrons depending on their state of valence. ${ }^{23}$ Thus, in the absence of an electric field, the negatively charged environment leads to a luminescence spectrum dominated by negatively charged exciton complexes. ${ }^{24}$ When a bias is applied in either direction, these electrons are swept by the electric field, leading to neutral or positively charged exciton recombination.

Together with the external field, the local field associated with ionized defects ${ }^{12,25}$ and the screening field created by 
the accumulation of photogenerated carriers in the metalsemiconductor interfaces ${ }^{21}$ must be considered. The local field fluctuates due to the dynamics of the charged environment. ${ }^{25}$ This broadens the emission lines by spectral diffusion, as shown in Fig. 2(c) for the spectral line P1 (FWHM $\sim 800 \mu \mathrm{eV}$ ). The fluctuations are largely minimized once the extra charges have been swept, and switching to spectral lines P2-P4 (FWHM $\sim 110-260 \mu \mathrm{eV}$ ) takes place. Meanwhile, the screening is likely responsible for small energy shifts which we observe when varying the excitation power at constant bias [Fig. 2(c)]. Both effects are small, typically 100-500 $\mu \mathrm{eV}$, and can be disregarded for the analysis of the charge tuning effect where large shifts (1-8 meV), associated with carrier-carrier interactions, are induced by the external bias. Both effects can be also minimized by using a resonant excitation scheme. ${ }^{12}$

The charge tunability is crucial for applications in quantum information technology and also to identify the different spectral lines in our experiment. The tunneling rates are determined by the carrier confinement energies and therefore depend on the QD size and the Coulomb interactions between electrons and holes. ${ }^{22,26}$ This leads to $\mu$-PL contour maps with characteristic staircase patterns and energy splittings which are different for single QDs and QD pairs.

The four spectral lines (P1-P4) in the spectrum of Fig. 2(b) can be well described assuming that QN1 is a single QD. To do so, the electronic structure has been calculated using a two-dimensional (2D) effective mass model for electrons and heavy holes. ${ }^{20}$ We calculate the emission energy of the neutral exciton $\left(X^{0}\right)$, negative and positive trions $\left(X^{-}, X^{+}\right)$, the biexciton $\left(X X^{0}\right)$, and the negative quarton $\left(X^{2-}\right)$ [see the inset of Fig. 2(b)]. By comparing the energetic ordering with that of the experiment, we find that lines P1, P2, P3, and P4 correlate well with $X^{-}, X X^{0}, X^{0}$, and $X^{+}$optical transitions, respectively. The $X^{2-}$ triplet resonance, which would show up at lower energies, is not observed in the spectrum, indicating that only one electron is being transferred from the environment to this particular QD.

AFM performed in uncapped samples reveals that most of the nanoholes contain lateral QD pairs. Accordingly, most of our spectra cannot be described assuming just a single QD. In Fig. 3, we analyze in detail the spectrum of QN2, which is characterized by twice the number of spectral lines expected for a single QD. 7,8,28,29 The spectrum of QN2 can be understood by the tentative assignment proposed in the figure, which is roughly that of two single QDs, $A$ and $B$, with emission from $X^{2-}, X^{-}, X^{0}$, and $X^{+}$. The two QDs are asymmetric, as usually observed in AFM, with $A$ being slightly bigger. Starting from the $X^{2-}$ transitions (singlet and triplet), with increasing external field, the number of additional electrons is tuned from two to zero, and each QD in the pair follows its own Coulomb staircase: $X^{2-} \rightarrow X^{-} \rightarrow X^{0} \rightarrow X^{+}$. This shows that net confined charge can be controlled in a QD pair by applying a lateral electric field.

The energy ordering in the spectral assignment of Fig. 3 is the usual one for isolated QDs. Yet, there is a remarkable anomaly. The emission energy of $X_{A}^{0}$ is lower than that of $X_{B}^{0}$, indicating that $\mathrm{QD} A$ is bigger than QD $B$. However, the emission energy of $X_{A}^{-}$is higher than that of $X_{B}^{-}$. This result is difficult to explain in terms of isolated QDs. If we assume that the different structural conditions of the QDs lead to lower

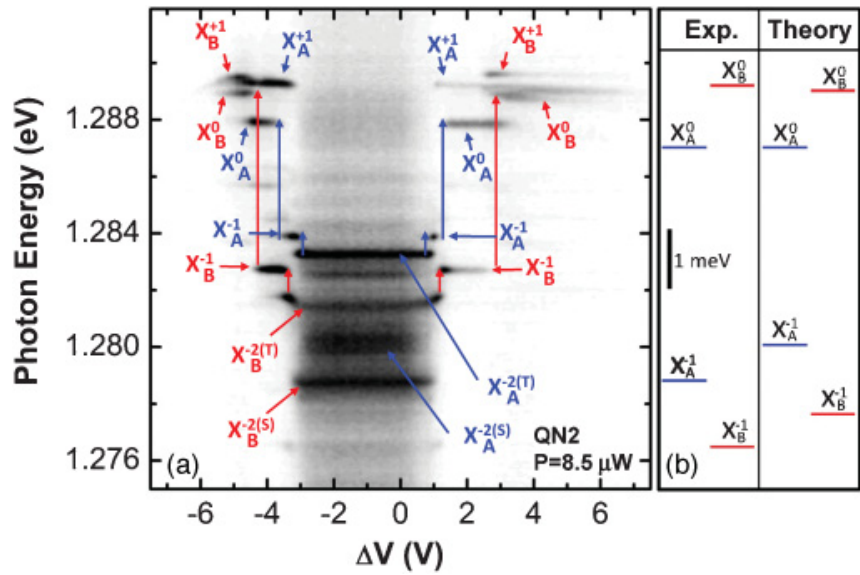

FIG. 3. (Color online) (a) $\mu$-PL contour plot map vs lateral bias measured for QN2. (b) Comparison between experimental (left-hand side) and theoretical (right-hand side) energies for $X^{0}$ and $X^{-}$ emission from QD A and B $(d=30 \mathrm{~nm})$.

energy for $X_{A}^{0}$, they should also lead to lower energy for $X_{A}^{-} \cdot{ }^{29}$ The anomalous behavior can be explained if the electrons in the QD pair are tunnel coupled. To illustrate this point, in Fig. 3(b) we compare the experimental energies of neutral and negatively charged excitons (left-hand column) with those of a coupled QD pair (right-hand column). The QDs have the same parameters as in the single QD case of Fig. 2, but QD $A$ is now slightly bigger $\left(\hbar \omega_{e}=34 \mathrm{meV}\right)$. To enable tunnel coupling, we consider the distance between the centers of the QDs is $d=30 \mathrm{~nm}$, which is slightly below the average value found by AFM. Under these conditions, not only is the calculated energy ordering the same as in the experiment, but also we obtain remarkable agreement in the energy splittings. In the experiment, the $X^{0}$ peaks are split by $1.06 \mathrm{meV}$ and the $X^{-}$ peaks by $-1.13 \mathrm{meV}$. In the calculations, the corresponding values are 0.96 and $-1.17 \mathrm{meV}$, respectively.

To understand the reversed ordering of the trion emission lines as compared to that of excitons, one must notice the different response of the two species when the QD pair is

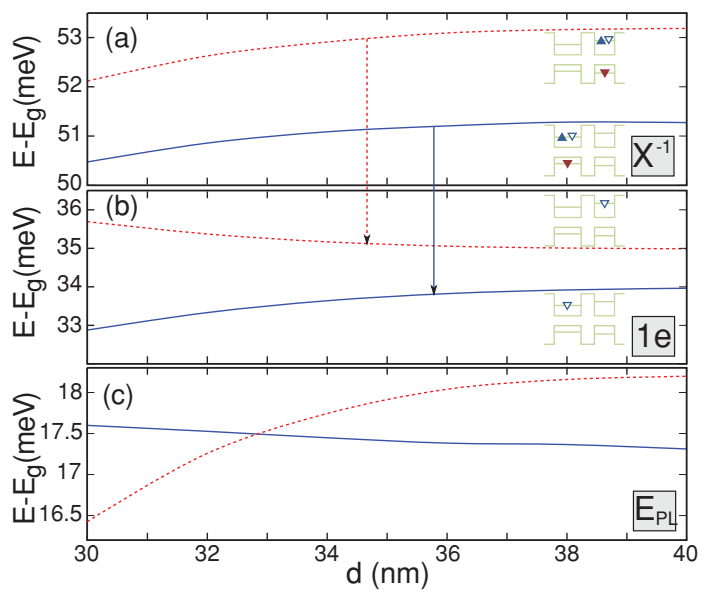

FIG. 4. (Color online) Calculated energy of the negative trions (a), electrons (b), and corresponding trion emission line (c) as a function of the interdot distance $d$. The insets indicate the location of electrons and holes in the long $d$ limit. $E_{g}$ is the gap energy. 
approached. In asymmetric QD pairs, the energy splitting between the two direct $X^{0}$ states $\left(X_{A}^{0}\right.$ and $\left.X_{B}^{0}\right)$ is barely sensitive to the interdot distance, up to very small separations. ${ }^{30}$ The situation is similar for $X^{-}$, as shown in Fig. 4(a). However, the final electron states display a pronounced tunnel coupling, ${ }^{31}$ which translates into a sizable energy splitting with decreasing interdot distance [see Fig. 4(b)]. In particular, the electron in QD $B$ evolves toward an antibonding molecular state. As a consequence, the emission energy $\left[E_{\mathrm{PL}}\left(X^{-}\right)=\right.$ $\left.E\left(X^{-}\right)-E\left(e^{-}\right)\right]$of the trion in QD $B$ decreases and eventually crosses that of QD $A$ at $d \sim 33 \mathrm{~nm}$, as shown in Fig. 4(c). A further reduction in the interdot distance results in reversed ordering of our experimental spectra.

To conclude, we have shown how the number of electrons can be electrically controlled in lateral QD pairs embedded in a lateral Schottky diode. As the number of confined electrons is lowered, carrier-carrier interactions give rise to well-defined energy shifts, which we compare with a theoretical model including Coulomb interactions and electron tunnel coupling. We have found that the negative trion emission energy is sensitive to single electron tunneling for interdot distances $d<40 \mathrm{~nm}$. These results are relevant in the field of scalable quantum information processing using laterally coupled QDs.

The authors wish to acknowledge to Spanish MICINN through projects Consolider-Ingenio 2010 QOIT (CSD20060019), TEC2008-06756-C03-01/03, and CTQ2008-03344, and to Comunidad Autónoma de Madrid through project Q\&CLight (S2009ESP-1503). *benito.alen@imm.cnm.csic.es

${ }^{1}$ L. Robledo, J. Elzerman, G. Jundt, M. Atatüre, A. Högele, S. Fält, and A. Imamoglu, Science 320, 772 (2008).

${ }^{2}$ D. Kim, S. E. Economou, S. C. Badescu, M. Scheibner, A. S. Bracker, M. Bashkansky, T. L. Reinecke, and D. Gammon, Phys. Rev. Lett. 101, 236804 (2008).

${ }^{3}$ D. Kim, S. G. Carter, A. Greilich, A. S. Bracker, and D. Gammon, Nat. Phys. 7, 223 (2011)

${ }^{4}$ H. J. Krenner, M. Sabathil, E. C. Clark, A. Kress, D. Schuh, M. Bichler, G. Abstreiter, and J. J. Finley, Phys. Rev. Lett. 94, 057402 (2005).

${ }^{5}$ E. A. Stinaff, M. Scheibner, A. S. Bracker, I. V. Ponomarev, V. L. Korenev, M. E. Ware, M. F. Doty, T. L. Reinecke, and D. Gammon, Science 311, 636 (2006).

${ }^{6}$ M. Scheibner, I. V. Ponomarev, E. A. Stinaff, M. F. Doty, A. S. Bracker, C. S. Hellberg, T. L. Reinecke, and D. Gammon, Phys. Rev. Lett. 99, 197402 (2007).

${ }^{7}$ G. J. Beirne, C. Hermannstädter, L. Wang, A. Rastelli, O. G. Schmidt, and P. Michler, Phys. Rev. Lett. 96, 137401 (2006).

${ }^{8}$ L. Wang, A. Rastelli, S. Kiravittaya, M. Benyoucef, and O. G. Schmidt, Adv. Mater. (Weinheim, Ger.) 21, 2601 (2009).

${ }^{9}$ J. Peng and G. Bester, Phys. Rev. B 82, 235314 (2010).

${ }^{10}$ P. Alonso-Gonzalez, J. Martin-Sanchez, Y. Gonzalez, B. Alen, D. Fuster, and L. Gonzalez, Cryst. Growth Des. 9, 2525 (2009).

${ }^{11}$ E. S. Moskalenko, M. Larsson, W. V. Schoenfeld, P. M. Petroff, and P. O. Holtz, Phys. Rev. B 73, 155336 (2006).

${ }^{12}$ E. S. Moskalenko, M. Larsson, K. F. Karlsson, P. O. Holtz, B. Monemar, W. V. Schoenfeld, and P. M. Petroff, Nano Lett. 7, 188 (2007).

${ }^{13}$ S. Volk, F. J. R. Schulein, F. Knall, D. Reuter, A. D. Wieck, T. A. Truong, H. Kim, P. M. Petroff, A. Wixforth, and H. J. Krenner, Nano Lett. 10, 3399 (2010).

${ }^{14}$ S. Volk, F. Knall, F. J. R. Schulein, T. A. Truong, H. Kim, P. M. Petroff, A. Wixforth, and H. J. Krenner, Appl. Phys. Lett. 98, 023109 (2011).

${ }^{15}$ K. Kowalik, O. Krebs, A. Lemaître, S. Laurent, P. Senellart, P. Voisin, and J. A. Gaj, Appl. Phys. Lett. 86, 041907 (2005).
${ }^{16}$ V. Stavarache, D. Reuter, A. D. Wieck, M. Schwab, D. R. Yakovlev, R. Oulton, and M. Bayer, Appl. Phys. Lett. 89, 123105 (2006).

${ }^{17}$ B. D. Gerardot, S. Seidl, P. A. Dalgarno, R. J. Warburton, D. Granados, J. M. Garcia, K. Kowalik, O. Krebs, K. Karrai, A. Badolato et al., Appl. Phys. Lett. 90, 041101 (2007).

${ }^{18}$ M. E. Reimer, W. R. McKinnon, J. Lapointe, D. Dalacu, P. Poole, G. C. Aers, D. Kim, M. Korkusinski, P. Hawrylak, and R. L. Williams, Physica E 40, 1790 (2008).

${ }^{19}$ B. Alén, D. Fuster, I. Fernández-Martínez, J. Martínez-Pastor, Y. González, F. Briones, and L. González, Nanotechnology 20, 475202 (2009).

${ }^{20}$ See Supplementary Material at http://link.aps.org/supplemental/ 10.1103/PhysRevB.84.041308 for a description of the calculations and evolution of spectral switching with power and temperature.

${ }^{21}$ C. Wei, H. Klein, and H. Beneking, Electron. Lett. 17, 688 (1981).

${ }^{22}$ B. Alén, J. Martínez-Pastor, D. Granados, and J. M. García, Phys. Rev. B 72, 155331 (2005).

${ }^{23}$ K. Laasonen, R. M. Nieminen, and M. J. Puska, Phys. Rev.B 45, 4122 (1992).

${ }^{24}$ P. Alonso-González, B. Alén, D. Fuster, Y. González, and L. González, Appl. Phys. Lett. 91, 163104 (2007).

${ }^{25}$ H. Kamada and T. Kutsuwa, Phys. Rev. B 78, 155324 (2008).

${ }^{26}$ R. J. Warburton, C. Schäflein, D. Haft, F. Bickel, A. Lorke, K. Karrai, J. M. García, W. Schoenfeld, and P. M. Petroff, Nature (London) 405, 926 (2000).

${ }^{27}$ R. Pomraenke, C. Lienau, Y. I. Mazur, Z. M. Wang, B. Liang, G. G. Tarasov, and G. J. Salamo, Phys. Rev. B 77, 075314 (2008).

${ }^{28}$ M. Abbarchi, C. A. Mastrandrea, A. Vinattieri, S. Sanguinetti, T. Mano, T. Kuroda, N. Koguchi, K. Sakoda, and M. Gurioli, Phys. Rev. B 79, 085308 (2009).

${ }^{29}$ J. I. Climente, A. Bertoni, and G. Goldoni, Phys. Rev. B 78, 155316 (2008).

${ }^{30}$ M. Baira, L. Sfaxi, L. Bouzaiene, H. Maaref, N. Chauvin, and C. Bru-Chevallier, J. Appl. Phys. 104, 064314 (2008).

${ }^{31}$ J. Peng, C. Hermannstädter, M. Witzany, M. Heldmaier, L. Wang, S. Kiravittaya, A. Rastelli, O. G. Schmidt, P. Michler, and G. Bester, Phys. Rev. B 81, 205315 (2010). 